TP Periodica Polytechnica Chemical Engineering

59(3), pp. 236-242, 2015

DOI: $10.3311 /$ PPch.7598

Creative Commons Attribution (i)

RESEARCH ARTICLE

\section{Sizing Problem of Intermediate Storages under Stochastic Operational Conditions}

\author{
Éva Orbán-Mihálykó ${ }^{1}$, Csaba Mihálykó ${ }^{1}$
}

Received 03 July 2014; accepted after revision 29 September 2014

\begin{abstract}
The operation of intermediate storages is investigated in this paper. The input process is supposed to be a batch process and the output process is assumed to be a continuous one. The operational conditions in the input process are stochastic with respect to time and the amount of material. The goal is the determination of the required size of the buffer to a given reliability. For the solution, an auxiliary function is introduced and an integral equation is set up for it. Its analytical solution is presented in a special case. In general cases we present the approximation of the reliability with the help of a hyperbolic tangent family. We compare the exact and approximate solutions and present the solution of the sizing problem. We investigate the effects of dispersions to present the uncertainties.
\end{abstract}

\section{Keywords}

intermediate storage, stochastic model, reliability, size
${ }^{1}$ Department of Mathematics, University of Pannonia,

H 8201 Veszprém, Egyetem u. 10., Hungary

${ }^{*}$ Corresponding author, e-mail: orbane@almos.uni-pannon.hu

\section{Introduction}

Intermediate storages are often used in industry, such as chemical, pharmaceutical, food industry, environmental industry, energy production, and so on [1]. Detailed possibilities can be found in [2]. They serve as a buffer which is able to balance the differences between the turnout and the usage of the material due to some uncertainties, maintenances, failures and so on. But in general sense banks, insurance companies serve as intermediate storages as well. They collect money, while in the industry material is usual. In this paper we use the industrial motivation and terminology. The assumptions concerning the operation correspond distinctive properties of the models. These assumptions demand different toolkits during investigation. Some techniques like Monte Carlo simulation is often applied, but mathematical methods can be very useful as well. The combination of mathematical tools and informatics seems to be the most effective nowadays in engineering $[3,4]$. The mathematical apparatus can be usually applied only to a certain extent, but it is useful for verifying the tools applied in computer engineering. We followed the mentioned way previously. Determination of the necessary initial amount was investigated earlier in the continuous model in [5], and in the discrete model in [6].

\section{The investigated model}

During this investigation the intermediate storage connects two types of processing subsystem, the input subsystem and the output one. The operation of the input subsystem is supposed to be a batch process while the operation of the output subsystem is continuous.

The input process produces the material and fills it into the buffer and the output subsystem withdraws it for further processing. A schematic figure of this system can be seen in Fig.1.

The operation of the batch subsystem is assumed to be random. The fillings happen at random time points and the amount of the filled material is random as well.

The time between the $i^{\text {th }}$ and $(i+1)^{\text {th }}$ filling is denoted by $t_{i}$, $\mathrm{i}=1,2, \ldots$. We investigate the process from time 0 . The time of the $\mathrm{i}^{\text {th }}$ filling equals $\sum_{k=1}^{i} t_{k}, \mathrm{i}=1,2, \ldots$ We suppose that $\mathrm{t}_{\mathrm{i}}, \mathrm{i}=1,2, \ldots$ 


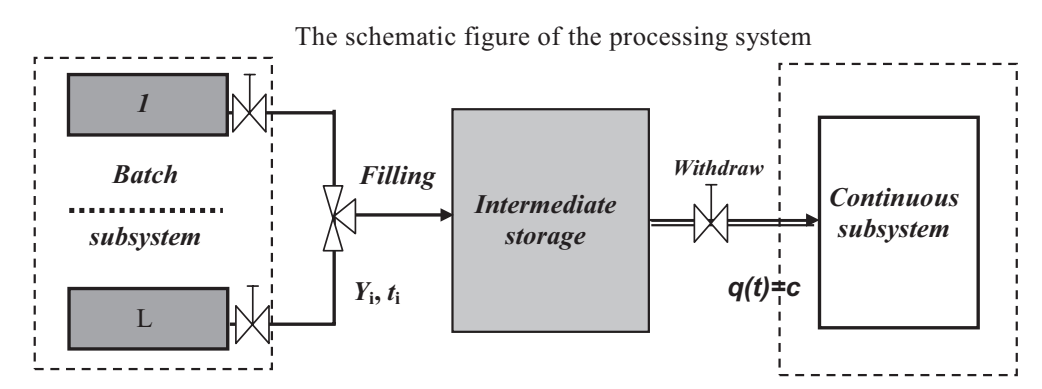

Fig. 1 Schematic model of a batch and continuous subsystem connected by an intermediate storage

are independent, identically distributed nonnegative continuous random variables with cumulative distribution function $F(t)$ and probability density function $\mathrm{f}(\mathrm{t})$. If $\mathrm{N}(\mathrm{t})$ denotes the number of fillings in the interval $[0, t], \mathrm{N}(\mathrm{t})$ can be expressed in the form

$$
N(\mathrm{t})=\left\{\begin{array}{llc}
0, & \text { if } & t<t_{1} \\
i, & \text { if } & \sum_{k=1}^{i} t_{k} \leq t<\sum_{k=1}^{i+1} t_{k} .
\end{array}\right.
$$

The amounts of material filled into the buffer during the fillings are described by identically distributed nonnegative continuous random variables with cumulative distribution function $\mathrm{G}(\mathrm{y})$ and probability density function $\mathrm{g}(\mathrm{y})$. The amount of material filled at the $i^{\text {th }}$ filling is denoted by $Y_{i} i=1,2, \ldots$. We suppose the independence of $Y_{i}$ ' $s$, moreover, the filling process $N(t)$ and filled material $Y_{i}$ are independent as well. The withdrawal is supposed deterministic and withdrawal rate is given as function of time $\mathrm{q}(\mathrm{t})$. Actually we deal with the case $\mathrm{q}(\mathrm{t})=\mathrm{c}$ positive constant value. Now, the material withdrawn from the buffer from time 0 to time $\mathrm{t}$ equals ct. The question is the size of the buffer in order to avoid overflow in a large time interval. We mention that due to the randomness the required size can be determined to a given reliability.

To solve this problem we investigate the amount of material in the intermediate storage as a function of time. Denoting the initial amount of material by $\mathrm{x}_{0}\left(0 \leq \mathrm{x}_{0}\right)$, the amount of material in the storage is

$$
U(t)=x_{0}+\sum_{i=1}^{N(t)} Y_{i}-c t
$$

If the size of the storage is $x_{\mathrm{s}}$, overflow means that the amount of material in the buffer exceeds the size of the storage, that is the inequality $x_{s}<x_{0}+\sum_{i=1}^{N(t)} Y_{i}-c t$ holds for some $0 \leq \mathrm{t}$.

As $x_{s}<U(t) \Rightarrow x_{s}-x_{0}<\sum_{i=1}^{N(t)} Y_{i}-c t$, overflow means that the maximal change of amount of material exceeds the difference $x_{s}-x_{0}$. Therefore, the distribution of the maximum value of the change is worth investigating. Denoting the difference $\mathrm{x}_{\mathrm{s}}-\mathrm{x}_{0}$ by $\mathrm{x}$, reliability means

$$
\Psi(x)=P\left(\left\{\sum_{i=1}^{N(t)} Y_{i}-c t \leq x \quad \forall t: 0 \leq t\right\}\right)
$$

and the probability of overflow equals $1-\Psi(\mathrm{x})$.
Time of overflow is the smallest time point, when overflow happens, that is

$$
T(x)=\left\{\begin{array}{l}
\inf \left\{\begin{array}{l}
t \geq 0: x<\sum_{i=1}^{N(t)} Y_{i}-c t, \text { if there } \\
\text { exists } t \geq 0: x<\sum_{i=1}^{N(t)} Y_{i}-c t
\end{array}\right. \\
\infty, \quad \text { if } \sum_{i=1}^{N(t)} Y_{i}-c t \leq x \text { for every } t \geq 0
\end{array}\right.
$$

A realization of the stochastic process which describes the amount of the material in the storage as a function of time can be seen in Fig. 2.

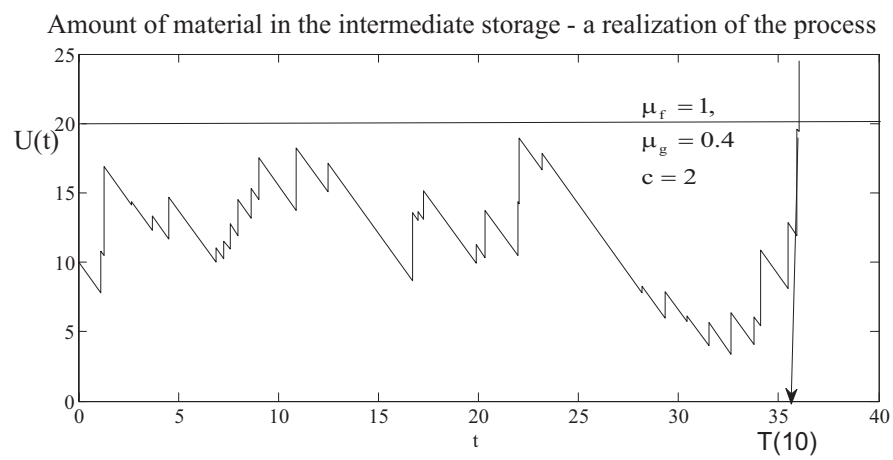

Fig. 2 A realization of the stochastic process describing the function of the amount of material in the storage

The initial amount of material was $x_{0}=10$, the size of the storage is $x_{s}=20$, their difference equals $x_{s}-x_{0}=10$. Interarrival times are exponentially distributed random variables with expectation $\mu_{\mathrm{f}}=1$, filled amount of material are also exponentially distributed with parameter $\mu_{\mathrm{g}}=4$. Withdrawal rate is $\mathrm{c}=2$. The downside lines show continuous withdraw and the upside jumps are the consequences of the random fillings. The first moment when the amount of material in the storage exceeds the size $\mathrm{x}_{\mathrm{s}}=20$ is about $\mathrm{T}(10) \approx 35.5$. This is the first time point when the inequality $20<\mathrm{U}(\mathrm{t})$ holds.

To investigate the reliability and the time of overflow it is worth investigating the following auxiliary function, which is called the Gerber-Shiu discounted penalty function in insurance mathematics [7]. Let

$$
m(x, \delta)=E\left(e^{-\delta T(x)} \cdot I_{T(x)<\infty}\right), \quad 0 \leq x, 0 \leq \delta,
$$


which is essentially the Laplace transform of the probability density function of the finite overflow time. One can easily see that

$$
m(x, 0)=1-\Psi(x)
$$

moreover

$$
\left.\frac{-\partial m(x, \delta)}{\partial \delta}\right|_{\delta=0}=E\left(T(x) \cdot I_{T(x)<\infty}\right) .
$$

Consequently the function $\mathrm{m}(\mathrm{x}, \delta)$ is appropriate for investigating both the reliability and the expected time of overflow.

\section{Integral equations and solutions}

In this part we present integral equations which are satisfied by the function $\mathrm{m}(\mathrm{x}, \delta)$ and we provide an analytical solution in a special case.

With the help of renewal theory the following theorems are proved:

Theorem 1 If the probability density function of the interarrival times is $f(t)$, and the probability density function of the filled amount of material is $\mathrm{g}(\mathrm{y})$, then, for any value $0 \leq \mathrm{x}$ and $0 \leq \delta, \mathrm{m}(\mathrm{x}, \delta)$ satisfies the following integral equation

$$
\begin{aligned}
m(x, \delta) & =\int_{0}^{\infty} \int_{0}^{x+c t} e^{-\delta t} m(x+c t-y, \delta) f(t) g(y) d y d t \\
& +\int_{0}^{\infty} \int_{x+c t}^{\infty} g(y) e^{-\delta t} f(t) d y d t
\end{aligned}
$$

Although we can not solve these equations in general, we can state the following:

Theorem 2 If $\mathrm{f}(\mathrm{t})=\mu_{\mathrm{f}} \exp \left(-\mu_{\mathrm{f}} \mathrm{t}\right) \quad 0<\mathrm{t}, \mathrm{g}(\mathrm{y})=\mu_{\mathrm{g}} \exp \left(-\mu_{\mathrm{g}} \mathrm{y}\right)$ $0<\mathrm{y}$, and $\mu_{\mathrm{f}} / \mu_{\mathrm{g}}<\mathrm{c}$, then

$$
m(x, \delta)=a(\delta) \exp (b(\delta) \cdot x),
$$

where

$$
a(\delta)=\frac{c \mu_{g}+\delta+\mu_{f}-\sqrt{\left(c \mu_{g}+\delta+\mu_{f}\right)^{2}-4 c \mu_{g}}}{2 c \mu_{g}}
$$

and

$$
b(\delta)=\frac{-c \mu_{g}+\delta+\mu_{f}-\sqrt{\left(c \mu_{g}+\delta+\mu_{f}\right)^{2}-4 c \mu_{g}}}{2 c} .
$$

Especially,

$$
1-\Psi(x)=m(x, 0)=\frac{\mu_{f}}{c \mu_{g}} \exp \left(-\frac{c \mu_{g}-\mu_{\mathrm{f}}}{c} \cdot x\right),
$$

and

$$
\begin{aligned}
E\left(T(x) \cdot I_{T(x)<\infty}\right)= & \left.\frac{-\partial m(x, \delta)}{\partial \delta}\right|_{\delta=0} \\
& =\left(\frac{\mu_{f}}{\left(c \mu_{g}-\mu_{f}\right) c \mu_{g}}+\frac{\mu_{f}^{2} x}{\left(c \mu_{g}-\mu_{f}\right) c^{2} \mu_{g}}\right) \\
& \exp \left(-\frac{c \mu_{g}-\mu_{\mathrm{f}}}{c} \cdot x\right) .
\end{aligned}
$$

Theorem 3 If the probability density function of the interarrival times is $\mathrm{f}(\mathrm{t})$ and $\mathrm{Y}_{\mathrm{i}}=1, \mathrm{i}=1,2, \ldots$ then, for any value $0 \leq \mathrm{x}$ and $0 \leq \delta, \mathrm{m}(\mathrm{x}, \delta)$ satisfies the following integral equation

$$
m(x, \delta)= \begin{cases}\int_{0}^{\infty} e^{-\delta t} m(x+c t-1, \delta) f(t) d t \quad \text { if } \quad 1 \leq x, \\ \int_{\frac{1-x}{c}}^{\infty} e^{-\delta t} m(x+c t-1, \delta) f(t) d t+\int_{0}^{\frac{1-x}{c}} e^{-t \delta} f(t) d t \text { if } \quad x<1\end{cases}
$$

The solution of the above equation in case of exponentially distributed inter-arrival times is a sum of polynomials and exponential functions. As the formula is complicated we omit it.

\section{Comparison of the analytical results and the simulated/approximate ones}

A possible method for the determination of the Gerber-Shiu function is Monte Carlo simulation. The simulation process is easy to realize. The main steps are the following:

- Fix the time interval $\left[0, \mathrm{~T}_{\max }\right]$ until the process is investigated, the value of the parameter $\mathrm{c}$ and the value of $\mathrm{x}$ and $\delta$. Fix the distribution of the inter-arrival times and filled amount of material by defining the probability density functions $f(t)$ and $g(y)$. Initialize the time point and initial amount of material as 0 .

- Generate random variables with probability density functions $f(t)$ and $g(y)$. Compute the time point of the actual filling and the amount of material being in the storage by summing the fillings and subtracting the withdrawn material. Check whether the amount exceeds the level $\mathrm{x}$. If yes, then overflow happens, the time of overflow is the actual time point. If not, repeat the activities of this point until the time passes the value $\mathrm{T}_{\max }$. If overflow does not happen, the value of $\mathrm{T}(\mathrm{x}) \cdot \mathrm{I}_{\mathrm{T}(\mathrm{x})<\infty}$ is defined as zero according to the definition. During this step we produce a realization of the process as it is presented in Fig. 2.

- Compute the actual value of $\mathrm{e}^{-\delta \mathrm{T}(\mathrm{x})} \cdot \mathrm{I}_{\mathrm{T}(\mathrm{x})<\infty}$.

- Repeat the simulation process several times. The expectation $\mathrm{E}\left(\mathrm{e}^{-\delta \mathrm{T}(\mathrm{x})} \cdot \mathrm{I}_{\mathrm{T}(\mathrm{x})<\infty}\right)$ is estimated by the average of the above actual values $\mathrm{e}^{-\delta \mathrm{T}(\mathrm{x})} \cdot \mathrm{I}_{\mathrm{T}(\mathrm{x})<\infty}$ arising from the realizations. 


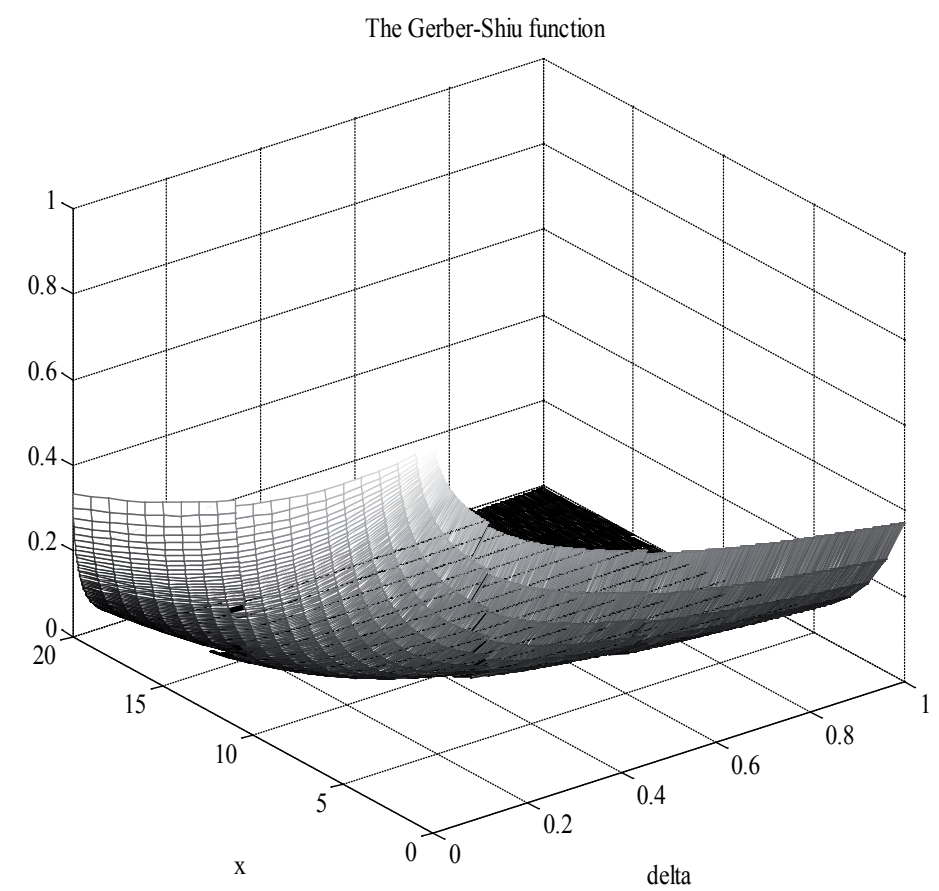

Fig. 3 Exact solution of the integral equation (8) in case of exponentially distributed inter-arrival time and filled amount of material

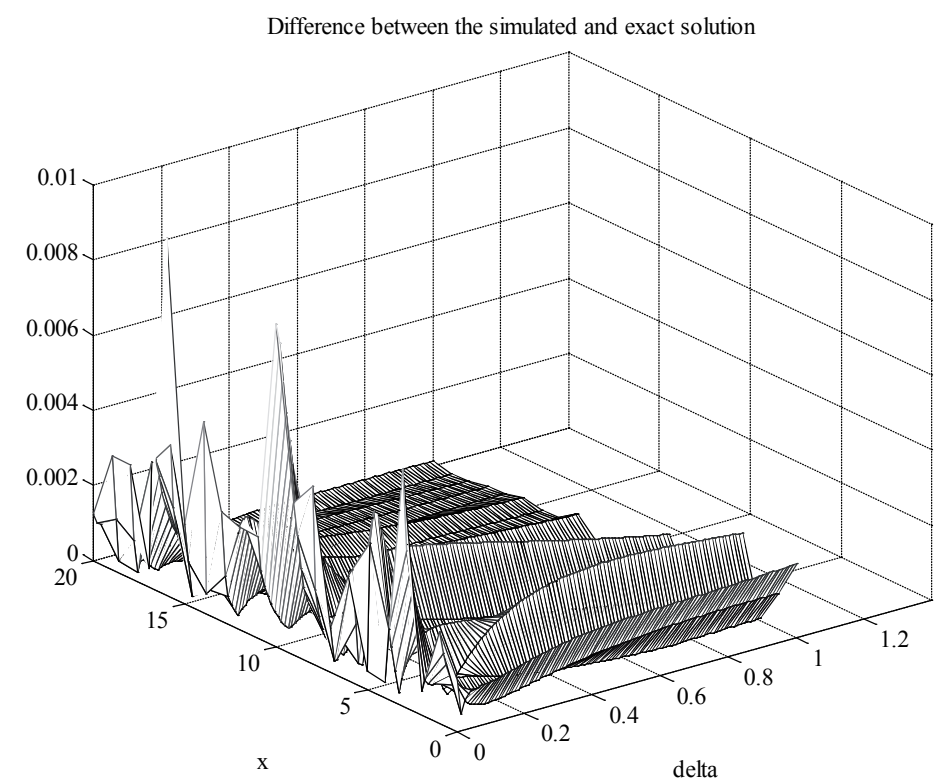

Fig. 4 Differences between the simulated and exact solutions of the integral equation (8) in case of exponentially distributed inter-arrival time and filled amount of material

In order to check the exactness of the simulation process we compare the simulated results and the exact ones given by (9). Fig.3. presents the exact values when the parameters are $\mu_{\mathrm{f}}=$ $1, \mu_{\mathrm{g}}=0.55, \mathrm{c}=2$.

Simulation was executed to $\mathrm{T}_{\max }=3000$, and the number of simulation was $\mathrm{N}=1000$.

The differences between the exact and the simulated values can be seen in Fig.4. and they are less than the error of the simulation. The parameters were the same as in case of Fig.3.

The probability of overflow as the function of the size can be seen in Fig.5. There are only small differences between the exact results and the simulations.

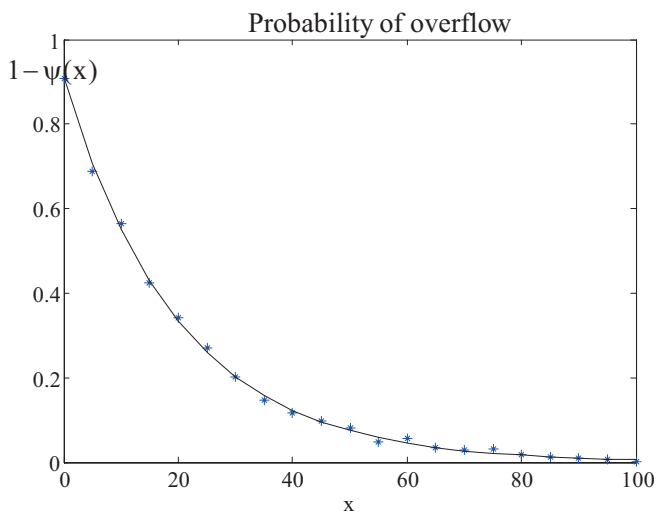

Fig. 5 Probability of overflow: exact (-) and simulated (*) results in case of exponentially distributed inter-arrival time and filled amount of material 


\section{Approximation of the reliability by parameter fitting}

The main relevance of the exact solution is the fact that one can try to find the solution in this form in general cases. Of course, we can have an approximate solution with this method. We can have simulation results and we can fit a function on them with the least squares method. We followed this way and we realized that the constant values of the exact solution can be approximated very well in the case of exponentially distributed inter-arrival times and filled amount of material. Moreover, the approximate coefficients are suitable for the approximation of the expected overflow time as well. While the exact function describing the expected overflow time is

$\mathrm{E}\left(\mathrm{T}(\mathrm{x}) \cdot \mathrm{I}_{\mathrm{T}(\mathrm{x})<\infty}\right)=(9.091+4.545 \cdot \mathrm{x}) \cdot \exp (-0.05 \cdot \mathrm{x})$ approximate one looks

$$
\begin{aligned}
E^{\text {app }}\left(T(x) \cdot I_{T(x)<\infty}\right) & =(10.581+4.298 \cdot \mathrm{x}) \\
& \cdot \exp (-0.048 \cdot \mathrm{x}) .
\end{aligned}
$$

As it can be seen in Fig. 6., their difference is quite small even in case of a small number of simulations $(\mathrm{N}=1000)$, which produces fluctuations in the values of simulated results.

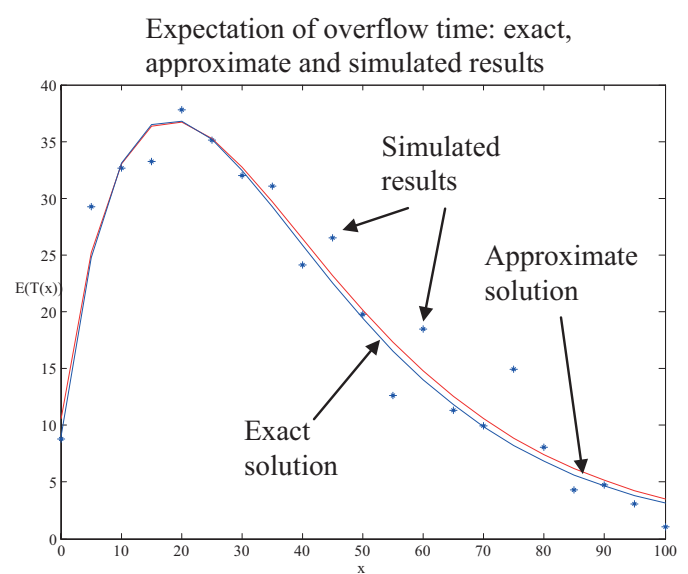

Fig. 6 Expectation of the overflow time- exact and approximate values and simulated points

In those cases when the exact solution is of other form, this approximation of reliability is less precise. Therefore we apply a more general function set for approximation. Let us approximate the exact solution in the following form

$$
\begin{gathered}
m^{a p p}(x, 0)=a \cdot\left(1-\tanh ^{m}\left(b x^{n}\right)\right), \\
\text { with } \\
0<a, 0<b, 0<n, 0<m .
\end{gathered}
$$

If we use this form, the exact solution is $\mathrm{m}(\mathrm{x}, 0)=0.9091$. $\exp (-0.05 \cdot \mathrm{x})$ while the approximate one is

$$
m^{a p p}(x, 0)=0.8961 \cdot\left(1-\tanh ^{1.6944}\left(0.1452 \mathrm{x}^{0.6449}\right)\right) .
$$

In Fig. 7 one can realize that the differences can hardly be seen.

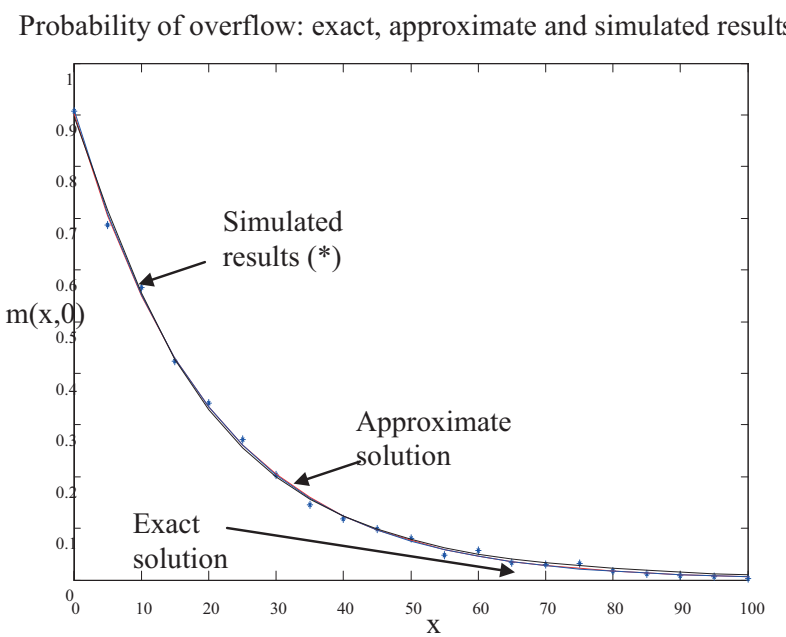

Fig. 7 Approximate and exact values of $m(x, 0)$ in case of exponentially distributed inter-arrival time and filled amount of material

The next figure presents the differences between the exponential-type approximation and the hyperbolic tangent approximation in case of Gauss distributed inter-arrival time and filled amount of material. The parameters were $\mathrm{E}\left(\mathrm{t}_{\mathrm{i}}\right)=1, \mathrm{D}\left(\mathrm{t}_{\mathrm{i}}\right)=0.1$, $\mathrm{E}\left(\mathrm{Y}_{\mathrm{i}}\right)=2, \mathrm{D}\left(\mathrm{t}_{\mathrm{i}}\right)=0.3, \mathrm{c}=3$. The end of the time interval was fixed $\mathrm{T}_{\max }=3000$, the number of simulations was $\mathrm{N}=10000$. One can see that in this case the hyperbolic tangent distribution family provides a better fitting to the simulated data than the exponential function.

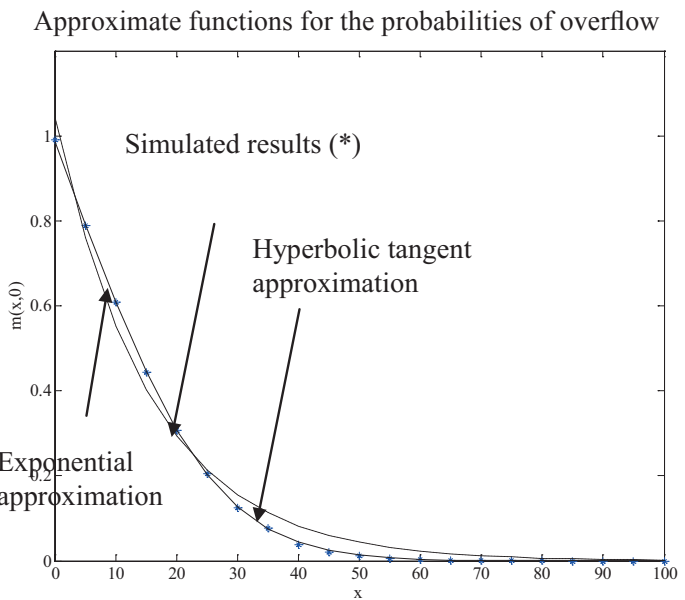

Fig. 8 Comparison of exponential (- . -) and hyperbolic tangent approximation (-) of overflow probabilities in case of Gauss distributed inter-arrival time and filled amount of material

The approximate function of exponential form is

$$
\mathrm{m}_{\text {exp }}^{\text {app }}(\mathrm{x}, 0)=1.0416(1-\exp (-0.0631 \mathrm{x}))
$$

while that of the hyperbolic tangent function is

$$
m_{\text {tanh }}^{a p p}(x, 0)=0.9645\left(1-\tanh ^{0.7320}\left(0.0143 \mathrm{x}^{1.2977}\right) .\right.
$$

Figure 8 represents that the hyperbolic tangent function fits better to the simulated points than the exponential function. The same phenomenon can be seen if the amount of the filled material is constant 1 . The inter-arrival time was Gauss 
distributed with $\mathrm{E}\left(\mathrm{t}_{\mathrm{i}}\right)=0.5, \mathrm{E}\left(\mathrm{t}_{\mathrm{i}}\right)=1, \mathrm{D}\left(\mathrm{t}_{\mathrm{i}}\right)=0.05, \mathrm{c}=2$. The approximate function of exponential form is

$$
\mathrm{m}_{\exp }^{\mathrm{app}}(\mathrm{x}, 0)=1.0439(1-\exp (-0.1593 \mathrm{x})),
$$

while that of the hyperbolic tangent function is

$$
m_{\text {tanh }}^{a p p}(x, 0)=0.9890\left(1-\tanh ^{0.8810}\left(0.0673 \mathrm{x}^{1.1934}\right) .\right.
$$

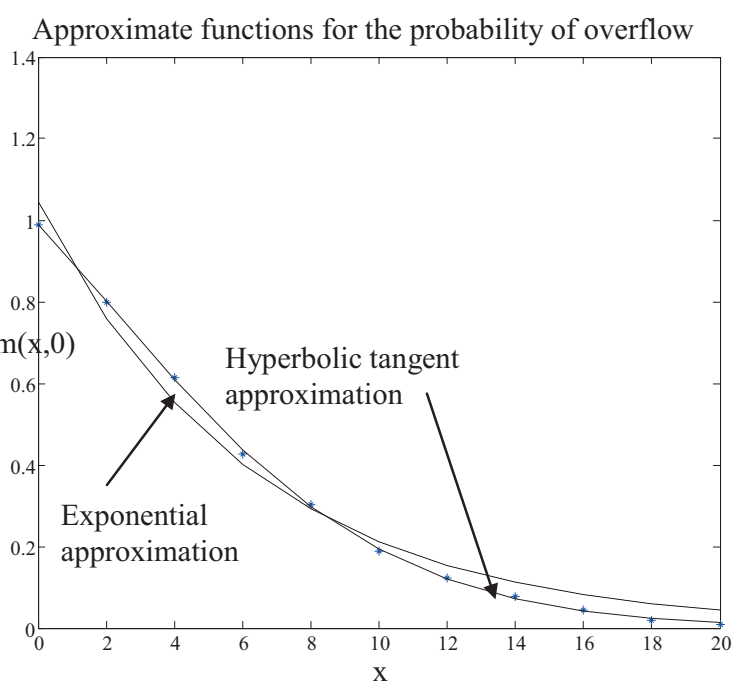

Fig. 9 Comparison of exponential and hyperbolic tangent approximation of overflow probabilities in case of Gauss distributed inter-arrival time and constant filled amount of material

\section{Determination of the required size of storage to a given reliability}

Actually let us return to the industrial problem, namely determine the size of the storage which is large enough to avoid the overflow with a given probability $1-\alpha$ (reliability). That is, after fixing the value of $1-\alpha$, we would like to know the value $\mathrm{x}$ for which

$$
P\left(\sum_{i=1}^{N(t)} Y_{i}-c t \leq x, \quad \forall t: 0 \leq t \leq T_{\max }\right)=1-\alpha .
$$

Now

$$
P\left(x<\sum_{i=1}^{N(t)} Y_{i}-c t, \quad \text { for some } t: 0 \leq t \leq T_{\max }\right)=\alpha,
$$

that is $\alpha$ is the overflow probability. For this purpose we determine the solution of the equation

$$
m^{a p p}(x, 0)=a\left(1-\tanh ^{m}\left(b x^{n}\right)=\alpha .\right.
$$

Arranging (24) we get

$$
x=\left(\frac{\operatorname{artanh}\left(1-\frac{\alpha}{a}\right)^{\frac{1}{m}}}{b}\right)^{\frac{1}{n}} .
$$

which is a closed form for the required size.
In case of Gauss distributed inter-arrival time and filled amount of material with the parameters of Fig. 8 the equation to solve is

$$
\begin{aligned}
m_{\tanh }^{a p p}(x, 0) & =0.9645\left(1-\tanh ^{0.7320}\left(0.0143 \cdot \mathrm{x}^{1.2977}\right)\right. \\
& =\alpha
\end{aligned}
$$

The solution is given by (25), the required sizes are plotted as the function of overflow probability in Fig. 10. The larger the probability, the smaller the size.

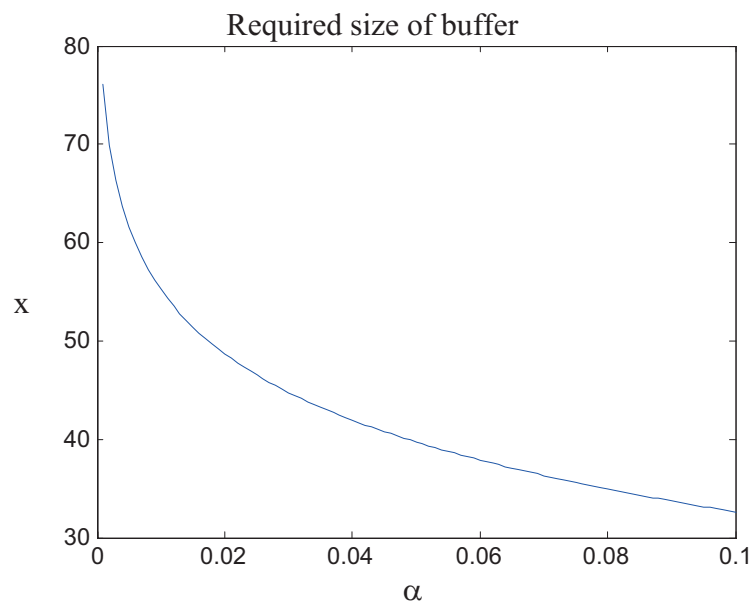

Fig. 10 Function (23) as a variable of the overflow probability $\alpha$

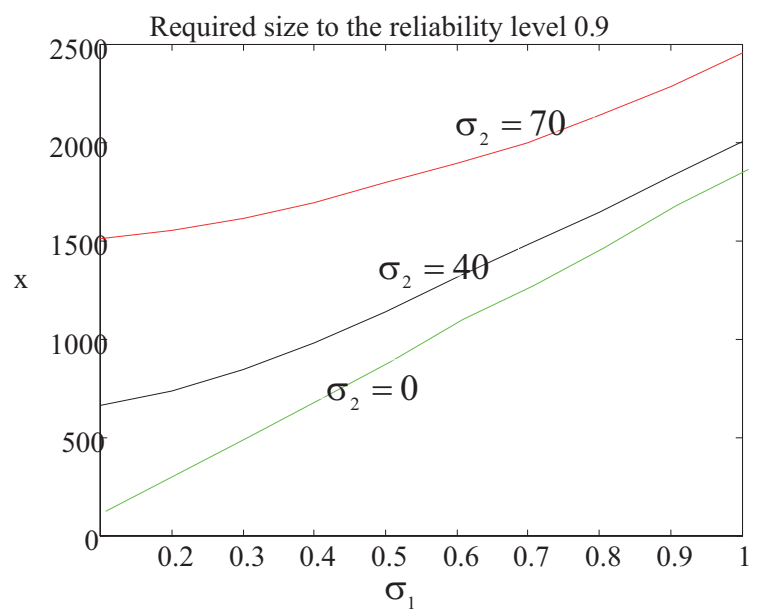

Fig. 11 Required size capacity as the function of $\sigma_{1}$ to the reliability level $1-$ $\alpha=0.9$ in case of different values of $\sigma_{2}$

\section{Effects of dispersions}

Finally we present the effects of the uncertainties on the required size of the buffer for a given reliability level. The following process arises from practice: some kind of chemical process needs an organic solvent. The solvent arrives in batches which are approximately Gauss distributed and the time points of arrival are Gauss distributed as well. The necessity of the solvent is $\mathrm{c}=87 \mathrm{~kg} / \mathrm{h}$. The expectation of inter-arrival time is $\mathrm{m}_{1}$ $=3.9667 \mathrm{~h}$, the dispersion varies from zero to 1 . The expectation of batches is $\mathrm{m}_{2}=345 \mathrm{~kg}$, the dispersion varies from 0 to 70. Figure 11 represents the required storage capacity to avoid overflow with reliability 0.9 (that is $\alpha=0.1$ ) to $\mathrm{T}_{\max }=720$ hours 


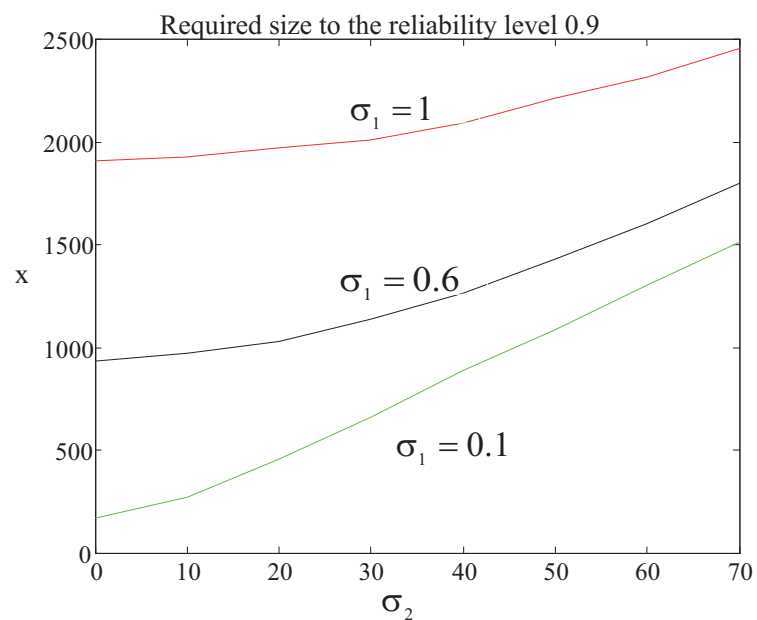

Fig. 12 Required size capacity to the reliability level $1-\alpha=0.9$ as the function of $\sigma_{2}$ in case of different values of $\sigma_{1}$

as the function of the dispersion of inter-arrival time in case of different values of dispersion of filled amount of material. Figure 11 represents that the larger the dispersion the larger the required size, as it is expected.

Figure 12 shows the required storage capacity to avoid overflow with reliability 0.9 (that is $\alpha=0.1$ ) to $\mathrm{T}_{\max }=720$ hours as the function of the dispersion of filled amount of material in case of different values of dispersion of inter-arrival time. Again, the larger the dispersion the larger the required size, as it is expected. We mention that $\sigma_{2}=0$ corresponds to the case of constant amount of filled material.

\section{Summary}

This paper deals with the determination of the required size of an intermediate storage to a given reliability level. An auxiliary function is introduced and an integral equation is presented for it. The exact solution is given for a special case and is compared to the simulated results. In general cases we suggest a family of functions for approximating the exact values of overflow probabilities and compare them to the exact solutions. The approximate function is applied to determine the required size.

\section{References}

[1] Hajnal, É., Almásy, G., Kollár-Hunek, K., Kollár, G. "Resource optimization by simulation technique in food logistics." Applied Ecology and Environmental Research. 5 (1). pp. 189-200. 2007.

DOI: 10.15666/aeer/0501_189200

[2] Special waste treatment. [Online] Available from: http://www.vega.com/ en/Environment_Recycling_Storage_Tanks_Intermediate_Storage_ Level.htm. [Accessed: 19th September 2014]

[3] Bárkányi, Á., Lakatos, B. G., Németh, S. "Modelling of heat exchange between drops in suspension polymerization of vinyl chloride." Periodica Polytechnica Chemical Engineering. 56 (2). pp. 55-64. 2012. DOI: 10.3311/pp.ch.2012-2.02

[4] Süle, Z., Lakatos, G. B., Mihálykó, Cs., Orbán-Mihálykó, É. "Modelling of Heat Transfer Processes with Compartment/Population Balance Model." Periodica Polytechnica Chemical Engineering. 57 (1-2). pp. 3-9. 2013. DOI: 10.3311/PPch.2163

[5] Orbán-Mihálykó, É., Mihálykó, Cs. "Investigation of operation of intermediate storages applying probability density functions satisfying linear differential equation." Periodica Polytechnica Chemical Engineering. 56 (2). pp. 77-82. 2012. DOI: 10.3311/pp.ch.2012-2.05

[6] Orbán-Mihálykó, É., Mihálykó, Cs. "Discrete Models for intermediate storages under stochastic operational conditions." Periodica Polytechnica Chemical Engineering. 58 (2). pp. 103-109. 2014. DOI: 10.3311/PPch.2192

[7] Gerber, H., Shiu, E. S. W. "On the time value of ruin." North American Actuarial Journal. 2 (1). pp. 48-78. 1998. DOI: $10.1080 / 10920277.1998 .10595671$ 